\title{
NUMERICAL SOLUTIONS OF NONSTANDARD FIRST ORDER INITIAL VALUE PROBLEMS ${ }^{1}$
}

\author{
M. VENKATESULU and P.D.N. SRINIVASU \\ Department of Mathematics \\ Sri Sathya Sai Institute of Higher Learning \\ Prasanthinilayam - 515134 \\ Andhra Pradesh \\ INDIA
}

\begin{abstract}
Differential equations of the form $y^{\prime}=f\left(t, y, y^{\prime}\right)$, where $f$ is not necessarily linear in its arguments, represent certain physical phenomena and are known for quite some time. The well known Clairut's and Chrystal's equations fall into this category. Earlier, we established the existence of a (unique) solution of the nonstandard initial value problem (NSTD IVP) $y^{\prime}=f\left(t, y, y^{\prime}\right), y\left(t_{0}\right)=y_{0}$ under certain natural hypotheses on $f$. In this paper we present some first order convergent numerical methods for finding the approximate solutions of the NSTD IVPs.
\end{abstract}

Key words: Nonstandard initial value problem, existence, unique solution, real valued, continuous, differentiable, numerical scheme, inequality, partition, approximate, algorithm, influence, round-off error, estimate, truncation.

AMS (MOS) subject classification: $\quad 34 \mathrm{~A} 99,34 \mathrm{~A} 50$.

\section{INTRODUCTION}

Differential equations of the form $y^{\prime}=f\left(t, y, y^{\prime}\right)$, where $f$ is not necessarily linear in its arguments, represent certain physical phenomena and are known for quite some time. The well known Clairut's and Chrystal's equations fall into this category [2]. A few authors, notably E.L. Ince [3], H.T. Davis [2], et. al. have given some methods for finding solutions of equations of the above type. In fact these methods are best described as follows.

If there exists $\left(t_{0}, y_{0}\right)$ such that the equation $y^{\prime}=f\left(t, y, y^{\prime}\right)$ can be solved for $y^{\prime}$ as a single valued function of $(t, y)$ in a neighborhood of $\left(t_{0}, y_{0}\right)$, say $y^{\prime}=g(t, y)$, then the solution of the initial value problem $(I V P) y^{\prime}=g(t, y), y\left(t_{0}\right)=y_{0}$, if it exists, is also a solution of the original equation $y^{\prime}=f\left(t, y, y^{\prime}\right)$ (and satisfies the initial condition $\left.y\left(t_{0}\right)=y_{0}\right)$. Or, if there exists $\left(t_{0}, y_{0}\right)$ such that the equation $y^{\prime}=f\left(t, y, y^{\prime}\right)$ can be solved for $y^{\prime}$ as a multivalued function of $(t, y)$ in a neighborhood of

\footnotetext{
${ }^{1}$ Received: February, 1990. Revised: February, 1991.
} 
$\left(t_{0}, y_{0}\right)$, then a (nonunique) solution of the IVP $y^{\prime}=f\left(t, y, y^{\prime}\right), y\left(t_{0}\right)=y_{0}$ is given by certain (not necessarily convergent) infinite series.

In our earlier paper [7], we have established the existence of a (unique) solution of the nonstandard IVP $y^{\prime}=f\left(t, y, y^{\prime}\right), y\left(t_{0}\right)=y_{0}$ under certain natural hypotheses on $f$, and have shown the continuous dependence of the solution on initial conditions, and parameters. The existence result that we established in [7] is of theoretical nature and it does not provide methods of finding the solutions explicitly either analytically or numerically. Our aim in this paper is to present numerical methods for finding the approximate solutions of the nonstandard IVPs.

Before proceeding to numerical methods, we introduce few notations and the nonstandard $I V P$. Let $R$ denote the real line, and let $R^{n}$ denote the $n$-dimensional real space where ' $n$ ' is a positive integer. Let $\left(t_{0}, y_{0}\right) \in R^{2}$ and let $D$ be a convex subset of $R^{3}$ defined by $D=\left\{(t, y, z) \in R^{3}|| t-t_{0}|\leq a| y-,y_{0}|<b| z \mid, \leq c\right\}$ where $a, b$ and $c$ are some positive constants. Let $f$ be a continuous real valued function defined on $D$.

Consider the nonstandard IVP (NSTD IVP)

$$
y^{\prime}=f\left(t, y, y^{\prime}\right), y\left(t_{0}\right)=y_{0}
$$

where $y^{\prime}$ denotes the derivative of $y$.

Definition: By a solution $y$ of the NSTD IVP (1), we mean a continuously differentiable real valued function $y(t)$ defined for $t \in I$, where $I$ is some interval of the real line containing the point $t_{0}$ such that

i) $\quad y\left(t_{0}\right)=y_{0}$

ii) the triplet $\left(t, y(t), y^{\prime}(t)\right) \in D$ for all $t \in I$, and

iii) $\quad y^{\prime}(t)=f\left(t, y(t), y^{\prime}(t)\right)$ holds for all $t \in I$.

In Section 2, we shall prove the existence of a unique higher order regular solution of the IVP(1). In Section 3, we shall present linearly convergent numerical schemes for constructing the solution of IVP(1). In Section 4, we shall illustrate our methods by solving some NSTD IVPs.

\section{EXISTENCE OF A UNIQUE (HIGHER ORDER REGULAR) SOLUTION OF THE IVP (1)}

Theorem 1: Suppose that the real valued function $f$ defined on $D$ satisfies the following conditions:

I) $f$ is ' $p$ ' times continuous differentiable with respect to $(t, y, z) \in D$ where $p \geq 1$ is an integer.

II) $|f(t, y, z)| \leq c$ for all $(t, y, z) \in D$, and 
III) $\left|\frac{\partial f}{\partial y}(t, y, z)\right| \leq k_{1}$, and $\left|\frac{\partial f}{\partial z}(t, y, z)\right| \leq k_{2}$ for all $(t, y, z) \in D$ where $k_{1}>0$ and $0 \leq k_{2}<1$ are constants.

Then the NSTD IVP (1) has a unique solution $y$ existing on the interval $\left[t_{0}-\alpha, t_{0}+\alpha\right]$, where $\alpha$ is any number such that $0<\alpha<\min \left(\frac{1-k_{2}}{k_{1}}, \frac{b}{c}, a\right)$. Moreover, the solution $y$ is ' $p+1$ ' times differentiable on $\left[t_{0}-\alpha, t_{0}+\alpha\right]$.

Proof: To prove the existence of a unique solution, it is enough to verify conditions (i) $-(i i i)$ of Theorem 1 [7]. Clearly conditions $(I)$ and $(I I)$ imply the conditions $(i)$ and (ii) of Theorem 1 [7]. To verify condition (iii), let $\left(t, y_{1}, z_{1}\right)$ and $\left(t, y_{2}, z_{2}\right) \in D$. Then by the mean value Theorem [1], we have that

$$
\begin{gathered}
\left|f\left(t, y_{1}, z_{1}\right)-f\left(t, y_{2}, z_{2}\right)\right| \leq\left|\frac{\partial f}{\partial y}(t, \hat{y}, \hat{z})\right|\left|y_{1}-y_{2}\right|+\left|\frac{\partial f}{\partial z}(t, \hat{y}, \hat{z})\right|\left|z_{1}-z_{2}\right| \\
\leq k_{1}\left|y_{1}-y_{2}\right|+k_{2}\left|z_{1}-z_{2}\right|(\text { by }(I I I))
\end{gathered}
$$

where $(t, \hat{y}, \hat{z})$ is a point on the line segment joining $\left(t, y_{1}, z_{1}\right)$ and $\left(t, y_{2}, z_{2}\right)$. Thus $f$ satisfies conditions $(i)-(i i i)$ of Theorem 1 [7], and hence the IVP (1) has a unique solution $y$ existing on the interval $\left[t_{0}-\alpha, t_{0}+\alpha\right]$, for every $\alpha$ such that $0<\alpha<\min \left(\frac{1-k_{2}}{k_{1}}, \frac{b}{c}, a\right)$. To show that $y$ is ' $p+1$ ' times continuously differentiable, we proceed as follows.

From the definition of a solution, it follows that $y^{\prime}$ is a continuous function on $\left[t_{0}-\alpha, t_{0}+\alpha\right]$ and we now claim that $y^{\prime \prime}$ exists on $\left[t_{0}-\alpha, t_{0}+\alpha\right]$ and is continuous function. For, let $t \in\left[t_{0}-\alpha, t_{0}+\alpha\right]$ and $h \neq 0$ be sufficiently small. Consider the difference quotient

$$
\frac{y^{\prime}(t+h)-y^{\prime}(t)}{h}=\frac{f\left(t+h, y(t+h), y^{\prime}(t+h)\right)-f\left(t, y(t), y^{\prime}(t)\right)}{h} .
$$

By the mean value theorem, there exists a point $(\tau, \eta, \varsigma)$ on the line segment joining $\left(t, y(t), y^{\prime}(t)\right)$ and $\left(t+h, y(t+h), y^{\prime}(t+h)\right)$ such that

$$
\frac{y^{\prime}(t+h)-y^{\prime}(t)}{h}=\frac{\partial f}{\partial t}(\tau, \eta, \varsigma)+\frac{\partial f}{\partial y}(\tau, \eta, \varsigma)\left(\frac{y(t+h)-y(t)}{h}\right)+\frac{\partial f}{\partial z}(\tau, \eta, \varsigma)\left(\frac{y^{\prime}(t+h)-y^{\prime}(t)}{h}\right) .
$$

But we have

$$
\left.\left|1-\frac{\partial f}{\partial z}(\tau, \eta, \varsigma)\right| \geq 1-\left|\frac{\partial f}{\partial z}(\tau, \eta, \varsigma)\right| \geq 1-k_{2}>0 \text { (by }(I I I)\right) .
$$

Therefore, from relation (2), we get that

$$
\frac{y^{\prime}(t+h)-y^{\prime}(t)}{h}=\left\{\frac{\partial f}{\partial t}(\tau, \eta, \varsigma)+\frac{\partial f}{\partial y}(\tau, \eta, \varsigma)\left(\frac{y(t+h)-y(t)}{h}\right)\right\} /\left[1-\frac{\partial f}{\partial z}(\tau, \eta, \varsigma)\right]
$$

Now, taking the limit as $h \rightarrow 0$, we get that

$$
\lim _{h \rightarrow 0} \frac{y^{\prime}(t+h)-y^{\prime}(t)}{h}=\frac{\frac{\partial f}{\partial t}\left(t, y(t), y^{\prime}(t)\right)+\frac{\partial f}{\partial y}\left(t, y(t), y^{\prime}(t)\right) y^{\prime}(t)}{1-\frac{\partial f}{\partial z}\left(t, y(t), y^{\prime}(t)\right)}
$$


We observe that the right hand side of (4) is a well defined quantity and is a continuous function of t. Hence

$$
y^{\prime \prime}(t)=\frac{\frac{\partial f}{\partial t}\left(t, y(t), y^{\prime}(t)\right)+\frac{\partial f}{\partial y}\left(t, y(t), y^{\prime}(t)\right) y^{\prime}(t)}{1-\frac{\partial f}{\partial z}\left(t, y(t), y^{\prime}(t)\right)} \text { exists and is continuous. }
$$

Now, from condition $(I)$ and inequality (3), it follows that the right hand side of (5) is a ' $p-1$ ' times continuously differentiable function of ' $t$ ' on $\left[t_{0}-\alpha, t_{0}+\alpha\right]$, and hence the solution $y$ is ' $p+1$ ' times continuously differentiable on $\left[t_{0}-\alpha, t_{0}+\alpha\right]$.

\section{NUMERICAL SCHEMES FOR THE SOLUTION OF IVP (1)}

We assume that the conditions of Theorem 1 be satisfied and $p=1$. For each positive integer ' $n$ ', we divide the interval $\left[t_{0}, t_{0}+\alpha\right]$ into ' $n$ ' subintervals of equal length, i.e., we take a partition of the form $t_{0}<t_{1}<\ldots<t_{n-1}<t_{n}=t_{0}+\alpha$, where $t_{i}=t_{0}+i h, h=\alpha / n, i=0,1, \ldots, n$. For $t \in\left(t_{0}, t_{0}+\alpha\right)$ and $h$ sufficiently small such that $t+h \in\left[t_{0}, t_{0}+\alpha\right]$, by Taylor's theorem, the solution $y$ of the IVP (1) satisfies the relation

$$
y(t+h)=y(t)+h y^{\prime}(t)+\frac{h^{2}}{2} y^{\prime \prime}(t+\theta h), 0<\theta<1 .
$$

From (5) and (3), and condition (I), we easily get that $\left|y^{\prime \prime}(t)\right| \leq N$, for all $t \in\left[t_{0}-\alpha, t_{0}+\alpha\right]$ where $N>0$ is a constant.

Let $\eta_{i}$ denote the approximate value of $y$ at $t_{i}, i=0,1, \ldots, n$, and let $\epsilon$ denote tolerance limit.

\section{Scheme I (Contraction-Euler Method):}

We define

a) $\eta_{0}=y_{0}$

$$
\begin{aligned}
& \text { for } i=0,1,2, \ldots, n-1 \\
& \eta^{\prime}{ }_{i 0}=0 ; j=0
\end{aligned}
$$

b) repeat

$$
\begin{aligned}
& j=j+1 \\
& \eta_{i j}^{\prime}=f\left(t_{i}, \eta_{i}, \eta_{i j-1}^{\prime}\right)
\end{aligned}
$$

until $k_{2}^{j}<|h|$ and $\left|\eta_{i j}^{\prime}-\eta_{i j-1}^{\prime}\right| \leq \epsilon$ are satisfied;

c) $\quad \eta_{i+1}=\eta_{i}+h \eta_{i j}^{\prime}$

$$
t_{i+1}=t_{i}+h \text {. }
$$

This algorithm computes the approximate value of $y\left(t_{i}\right)$ at $t_{i}=t_{0}+i h, i=1,2, \ldots, n$. 
To show the validity of the above algorithm, let $m$ be the smallest non-negative integer $j$ which satisfies the conditions $k_{2}^{j}<|h|$ and $\left|\eta^{\prime}{ }_{i j}-\eta^{\prime}{ }_{i j-1}\right| \leq \epsilon$, for a fixed ' $i$ '. We have

$$
\begin{aligned}
&\left|\eta_{0}-y_{0}\right|=0 \leq b \\
&\left|n_{1}-y_{0}\right|=\left|\eta_{1}-\eta_{0}\right|=h\left|\eta_{0 m}^{\prime}\right| \leq \frac{\alpha}{n} c \leq b \\
&\left.\mid \eta_{2}-y_{1}\right) \leq\left|\eta_{1}-y_{0}\right|+h\left|\eta_{1 m}^{\prime}\right| \leq \frac{c \alpha}{n}+\frac{c \alpha}{n} \leq b \\
& \cdot \cdot \\
& \cdot \cdot \\
&\left|\eta_{i+1}-y_{0}\right| \leq\left|\eta_{i}-y_{0}\right|+h\left|\eta_{i m}^{\prime}\right| \leq \frac{c \alpha i}{n}+\frac{c \alpha}{n} \leq b \\
&\left|\eta_{n}-y_{0}\right| \leq\left|\eta_{n-1}-y_{0}\right|+h\left|\eta_{(n-1) m}^{\prime}\right| \leq \frac{(n-1) c \alpha}{n}+\frac{c \alpha}{n}=c \alpha \leq b,
\end{aligned}
$$

since $\alpha$ is such that $0<\alpha<\min \left(\left(1-k_{2}\right) / k_{1}, b / c, a\right)$. Clearly, we have $\left|\eta_{i m}^{\prime}\right| \leq c$ for all $i=0,1, \ldots, n$.

Lemma 1: [6] If the numbers $\xi_{i}$ satisfy the estimates of the form $\left|\xi_{i+1}\right| \leq(1+\delta)\left|\xi_{i}\right|+B$, where $\delta>0, B>0, i=1, \ldots, q, \ldots$, then $\left|\xi_{q}\right| \leq e^{q \delta}\left|\xi_{0}\right|+\left(\frac{e^{q \delta}-1}{\delta}\right) B$, $q=1,2,3, \ldots$

Theorem 2 (Error estimate and Convergence of the Scheme I): Lete $_{i}=\eta_{i}-y\left(t_{i}\right)$, $i=0,1, \ldots, n$. Then $\left|e_{i}\right| \leq \hat{N} h\left(e^{\left(\alpha k_{1} /\left(1-k_{2}\right)\right)}-1\right)$, for $i=0,1, \ldots, n$, where $\hat{N}>0$ is a constant.

Proof: Clearly, we have $e_{0}=\eta_{0}-y_{0}=y_{0}-y_{0}$. For brevity, let $y_{i}=y\left(t_{i}\right)$ and consider

$$
\begin{gathered}
e_{i+1}=\eta_{i+1}-y_{i}+1 \\
=\eta_{i}+h \eta_{i m}^{\prime}-\left(y_{i}+h y^{\prime}\left(t_{i}\right)+\frac{h^{2}}{2} y^{\prime \prime}\left(t_{i}+\theta h\right)\right), 0<\theta<1, \\
\text { (by }(6) \text { and }(7)), \\
=\left(\eta_{i}-y_{i}\right)+h\left(\eta^{\prime}{ }_{i m}-y^{\prime}\left(t_{i}\right)\right)-\frac{h^{2}}{2} y^{\prime \prime}\left(t_{i}+\theta h\right)
\end{gathered}
$$

Therefore, we have that

$$
\left|e_{i+1}\right| \leq\left|e_{i}\right|+\frac{N h^{2}}{2}+h\left|\eta_{i m}^{\prime}-y^{\prime}\left(t_{i}\right)\right|,
$$

where $N$ is the bound for $y^{\prime \prime}$.

Also, we have that $\eta^{\prime}{ }_{i m}=f\left(t_{i}, \eta_{i}, \eta^{\prime}{ }_{i m-1}\right)$ and $f$, for a fixed $\left(t_{i}, \eta_{i}\right)$, is a contraction on the closed set $\{z \in R|| z \mid \leq c\}$ with the contraction constant ' $k_{2}$ '. Therefore, by the contraction mapping theorem [4], there exists a unique value $\eta_{i}^{\prime}$ such that

$$
\eta_{i}^{\prime}=f\left(t_{i}, \eta_{i}, \eta_{i}^{\prime}\right),\left|\eta_{i}^{\prime}\right| \leq c
$$

and

$$
\left|\eta_{i m}^{\prime}-\eta_{i}^{\prime}\right| \leq \frac{k_{2}^{m}}{1-k_{2}} c
$$


Moreover we have that

$$
\begin{gathered}
\left|\eta_{i}^{\prime}-y^{\prime}\left(t_{i}\right)\right|=\left|f\left(t_{i}, \eta_{i}, \eta_{i}^{\prime}\right)-f\left(t_{i}, y_{i}, y^{\prime}\left(t_{i}\right)\right)\right| \\
\leq k_{1}\left|\eta_{i}-y_{i}\right|+k_{2}\left|\eta_{i}^{\prime}-y^{\prime}\left(t_{i}\right)\right|
\end{gathered}
$$

which implies that

$$
\left|\eta_{i}^{\prime}-y^{\prime}\left(t_{i}\right)\right| \leq\left(\frac{k_{1}}{1-k_{2}}\right)\left|\eta_{i}-y_{i}\right|=\left(\frac{k_{1}}{1-k_{2}}\right)\left|e_{i}\right| .
$$

Thus in view of (10) and (11), from (8) we get that

$$
\begin{aligned}
\left|e_{i+1}\right| & \leq\left|e_{i}\right|+\frac{N h^{2}}{2}+h\left(\left|\eta_{i m}^{\prime}-\eta_{i}^{\prime}\right|+\left|\eta_{i}^{\prime}-y^{\prime}\left(t_{i}\right)\right|\right) \\
& \leq\left|e_{i}\right|+\frac{N h^{2}}{2}+h\left(\frac{k_{1}}{1-k_{2}}\left|e_{i}\right|+\frac{k_{2}^{m} c}{1-k_{2}}\right) \\
= & \left(1+\frac{k_{1} h}{1-k_{2}}\right)\left|e_{i}\right|+\frac{N h^{2}}{2}+\frac{h k_{2}^{m} c}{1-k_{2}}, \quad i=0,1, \ldots, n-1 .
\end{aligned}
$$

Hence by lemma 1, we get that

$$
\begin{gathered}
\left|e_{q}\right| \leq e^{\frac{q h k_{1}}{1-k_{2}}} e_{0}+\frac{e^{\frac{q h k_{1}}{1-k_{2}}}-1}{\frac{h k_{1}}{1-k_{2}}}\left[\left(\frac{N h^{2}}{2}+\frac{h k_{2}^{m} c}{1-k_{2}}\right)\right] \leq\left(\frac{e^{\frac{\alpha k_{1}}{1-k_{2}}}-1}{\frac{k_{1}}{1-k_{2}}}\right)\left(\frac{N h}{2}+\frac{k_{2}^{m} c}{1-k_{2}}\right)\left(\text { since } e_{0}=0\right) \\
=\hat{N} h\left(e^{\left(\alpha k_{1} /\left(1-k_{2}\right)\right)}-1\right), \text { where } \hat{N}=\left(\frac{N}{2}+\frac{c}{1-k_{2}}\right)\left(\frac{1-k_{2}}{k_{1}}\right) \quad\left(\text { since } k_{2}^{m}<|h|\right), \\
\text { for } q=1,2, \ldots, n .
\end{gathered}
$$

Influence of the round-off errors: Let $y_{i}=y\left(t_{i}\right)$ be the value of the exact solution $y$ of the $I V P(1), \eta_{i}$ be the approximate solution produced by the scheme $I$, and $\hat{\eta}_{i}$ be the approximate value of $\eta_{i}$ actually obtained in the digit floating point arithmetic, at $t_{i}$ for $i=0,1, \ldots, n$. Let $R_{i}=\hat{\eta}-\eta_{i}$ be the round-off error at the $i$-th step. Then we have

$$
\eta_{i+1}=\eta_{i}+h \eta_{i m}^{\prime}-R_{i+1}
$$

and

$$
y_{i+1}=y_{i}+h y^{\prime}\left(t_{i}\right)+\frac{h^{2}}{2} y^{\prime \prime}\left(t_{i}+\theta h\right) \text {. }
$$

Letting $e_{i}=y_{i}-\eta_{i}$, and subtracting (13) from (14), then in view of (12), we get

$$
\left|e_{i+1}\right| \leq\left(1+\frac{h k_{1}}{1-k_{2}}\right)\left|e_{i}\right|+\frac{N h^{2}}{2}+\frac{h k_{2}^{m} c}{1-k_{2}}+R_{i+1}
$$

Denoting $R=\max _{i=0,1, \ldots n} R_{i}$ and since $k_{2}^{m}<|h|$, we get from (15) that $\left|e_{i+1}\right| \leq\left(1+\frac{h k_{1}}{1-k_{2}}\right)$ $\left|e_{i}\right|+\left(\frac{N}{2}+\frac{c}{1-k_{2}}\right) h^{2}+R, i=0,1, \ldots, n-1$, and hence by lemma 1 , we get that

$$
\begin{gathered}
\left|e_{q}\right| \leq\left(\left(\frac{N}{2}+\frac{c}{1-k_{2}}\right) h^{2}+R\right)\left(\frac{e^{\frac{q h k_{1}}{1-k_{2}}}-1}{\frac{h k_{1}}{1-k_{2}}}\right) \leq\left(N_{1} h+\frac{R}{h}\right)\left(e^{\frac{\alpha k_{1}}{1-k_{2}}}-1\right)\left(\frac{1-k_{2}}{k_{1}}\right), \\
q=1,2, \ldots, n, \text { where } N_{1}=\frac{N}{2}+\frac{c}{1-k_{2}} .
\end{gathered}
$$


In the above inequality, $N_{1} h$ represents the truncation-contraction error term, and $R / h$ represents the round-off error term. We observe that as $h \rightarrow 0$, the truncation-contraction error term goes to zero where as the round-off error term becomes infinity. On the other hand, as $h \rightarrow+\infty$, the round-off error term goes to zero where as the truncation-contraction error term becomes infinitely large. Here, on account of the round-off errors, the total error begins to increase once again if $h$ is reduced beyond a certain critical value and that value is $h=\left(R / N_{1}\right)^{\frac{1}{2}}$.

Scheme II (Euler-Contraction Method):

We define

a) $\quad \eta_{0}=y_{0}$

$$
\begin{aligned}
& \text { for } i=0,1, \ldots, n-1, \\
& \qquad \eta_{i+1,0}=\eta_{i}, j=0
\end{aligned}
$$

b) repeat

$$
\begin{aligned}
& j=j+1 \\
& \eta_{i+1, j}=\eta_{i}+h f\left(t_{i}, \eta_{i}, \frac{\eta_{i+1, j-1}-\eta_{i}}{h}\right)
\end{aligned}
$$

c) until $k_{2}^{j}<|h|$ and $\left|\eta_{i+1, j}-\eta_{i+1, j-1}\right| \leq \epsilon$ are satisfied

$$
\begin{aligned}
& \eta_{i}=\eta_{i+1, j} \\
& t_{i+1}=t_{i}+h .
\end{aligned}
$$

As in the scheme I, the following theorem can be easily proved.

Theorem 3 (Error estimate and Convergence of the Scheme II): Let $e_{i}=\eta_{i}-y\left(t_{i}\right)$, $i=0,1,2, \ldots, n$. Then $\left|e_{i}\right| \leq \hat{N} h\left(e^{\alpha k_{1} /\left(1-k_{2}\right)}-1\right), i=0,1,2, \ldots, n$, where $\hat{N}$ is the same constant as in theorem 2.

From theorems 2 and 3 , it is clear that the numerical schemes I and II are equivalent in the sense that in both the cases the approximations converge to the true solution at the same rate. Also, it can be shown that the influence of the round-off errors in both the cases are same.

For the next two schemes to be developed below, we assume, in addition to the hypotheses of theorem 1 , the following conditions:

IV) $\frac{\partial^{2} f}{\partial z^{2}}(t, y, z)$ exists and continuous for $(t, y, z) \in D$, and hence $\left|\frac{\partial^{2} f}{\partial z^{2}}(t, y, z)\right| \leq k_{3}$ for all $(t, y, z) \in D$ where $k_{3}>0$ is a constant,

V) $\quad\left|\left[1-\frac{\partial f}{\partial z}(t, y, 0)\right]^{-1}\right| \leq k_{4}$ for all $(t, y, 0) \in D$, where $k_{4}>0$ is a constant such that $k_{3} k_{4} c<2 / 3$, and 
VI) $|f(t, y, 0)| \leq c_{1}$ for all $(t, y, 0) \in D$, where $c_{1}>0$ is a constant such that $c_{1} k_{4}<\left(1-\frac{3}{2} k_{3} k_{4} c\right) c$.

\section{Scheme III (Newton-Kantorovich-Euler Method):}

We define

a) $\quad \eta_{0}=y_{0}$

$$
\begin{aligned}
& \text { for } i=0,1,2, \ldots, n-1 \text {, } \\
& \eta_{i 0}^{\prime}=0 ; j=0
\end{aligned}
$$

b)

repeat

$$
\begin{aligned}
& j=j+1 \\
& \eta_{i j}^{\prime}=\eta_{i j-1}^{\prime}-\frac{\eta_{i j-1}^{\prime}-f\left(t_{i}, \eta_{i}, \eta_{i j-1}^{\prime}\right)}{1-\frac{\partial f}{\partial z}\left(t_{i}, \eta_{i}, \eta_{i j-1}^{\prime}\right)}
\end{aligned}
$$

until $\left(k_{3} k_{4} c / 6\left(1-k_{3} k_{4} c\right)\right)^{2^{j-1}}<h$ and $\left|\eta_{i j}^{\prime}-\eta_{i j-1}^{\prime}\right| \leq \epsilon$

are satisfied;

c)

$$
\begin{aligned}
& \eta_{i+1}=\eta_{i}+h \eta^{\prime}{ }_{i j} \\
t_{i+1}= & t_{i}+h .
\end{aligned}
$$

We note that the approximations $\eta^{\prime}{ }_{i m}$ are the Newton's approximations and by conditions $(I V)-(V I)$, they are well defined (see section 1.4 of [5]). The values $\eta_{i}$ are the Euler's approximations and are well defined as in scheme I.

Theorem \& (Error estimate and Convergence of the Scheme III): Let $e_{i}=\eta_{i}-y\left(t_{i}\right)$, $i=0,1,2, \ldots, n$. Then $\left|e_{i}\right| \leq \mathcal{N} h\left(e^{\alpha k_{1} /\left(1-k_{2}\right)}-1\right)$ where $\mathcal{N}>0$ is a constant.

Proof: Let $m$ denote the least non-negative integer such that the conditions $\left(k_{3} k_{4} c / 6\left(1-k_{3} k_{4} c\right)\right)^{2^{m-1}}<|h|$ and $\left|\eta_{i m}^{\prime}-\eta_{i m-1}^{\prime}\right| \leq \epsilon$ are true, for a fixed $i$. Clearly, we have $e_{0}=\eta_{0}-y_{0}=y_{0}-y_{0}=0$. For brevity, let $y_{i}=y\left(t_{i}\right)$ and consider

$$
\begin{gathered}
e_{i+1}=\eta_{i+1}-y_{i+1} \\
=\eta_{i}+h \eta_{i m}^{\prime}-\left(y_{i}+h y^{\prime}\left(t_{i}\right)+\frac{h^{2}}{2} y^{\prime \prime}\left(t_{i}+\theta h\right)\right), 0<\theta<1 \\
\text { (by (6) and (18)), }
\end{gathered}
$$

which implies that

$$
\left|e_{i+1}\right| \leq\left|e_{i}\right|+\frac{N h^{2}}{2}+h\left|\eta_{i m}^{\prime}-y^{\prime}\left(t_{i}\right)\right|
$$

where $N$ is the bound for $\left|y^{\prime \prime}\right|$.

Under the hypotheses of theorem 1 and conditions $(I V)-(V I)$, for each fixed $\left(t_{i}, \eta_{i}\right)$, $f\left(t_{i}, \eta_{i}, z\right)$ as a function of ' $z$ ' satisfies the conditions of theorem 1.4.5 [5] in the closed ball $\{z \in R|| z \mid \leq c\}$ and hence by theorem 1.4.5 and problem 1.4.8 of [5], there exists a unique value 
' $\eta_{i}^{\prime}$ ' such that

$$
\eta_{i}^{\prime}=f\left(t_{i}, \eta_{i}, \eta_{i}^{\prime}\right),\left|\eta_{i}^{\prime}\right| \leq c
$$

and

$$
\left|\eta_{i m}^{\prime}-\eta_{i}^{\prime}\right| \leq(\lambda / 3)^{2^{m-1}}(c / \lambda)
$$

where $\lambda=k_{3} k_{4} c / 2\left(1-k_{3} k_{4} c\right)(<3)$.

Now proceeding as in the proof of theorem 2, we get that

$$
\begin{gathered}
\left|e_{q}\right| \leq\left(e^{\frac{\alpha k_{1}}{1-k_{2}}}-1\right)\left(\frac{1-k_{2}}{k_{1}}\right)\left(\frac{N h}{2}+\left(\frac{\lambda}{3}\right)^{2^{m-1}} \frac{c}{\lambda}\right) \\
\leq \mathcal{N} h\left(e^{\frac{\alpha k_{1}}{1-k_{2}}}-1\right), \text { where } \mathcal{N}=\left(\frac{N}{2}+\frac{c}{\lambda}\right)\left(\frac{1-k_{2}}{k_{1}}\right) \text { (by (19)). }
\end{gathered}
$$

Influence of the round off errors: If we take the round-off errors into account, then as in the case of Scheme I, we get an estimate of the form $\left|e_{q}\right| \leq\left[\left(\frac{N}{2}+\frac{c}{\lambda}\right) h+\frac{R}{h}\right]\left(\frac{1-k_{2}}{k_{1}}\right)\left(e^{\frac{\alpha k_{1}}{1-k_{2}}}-1\right)$, $q=1,2, \ldots, n$, and hence the critical value for $h$ is given by $h=\left(R /\left(\frac{N}{2}+\frac{c}{\lambda}\right)\right)^{\frac{1}{2}}$.

Scheme IV (Euler-Newton-Kantorovich Method):

We define

a) $\quad \eta_{0}=y_{0}$

$$
\begin{aligned}
& \text { for } i=0,1,2, \ldots, n-1, \\
& \qquad \eta_{i+1,0}=\eta_{i} ; j=0
\end{aligned}
$$

b)

$$
\text { repeat }
$$

$$
\begin{aligned}
& j=j+1 \\
& \eta_{i+1, j}=\eta_{i+1, j-1}-\frac{\eta_{i+1, j-1}-\left(\eta_{i}+h f\left(t_{i}, \eta_{i}, \frac{\eta_{i+1, j-1}-\eta_{i}}{h}\right)\right)}{1-\frac{\partial f}{\partial z}\left(t_{i}, \eta_{i}, \frac{\eta_{i+1, j-1}-\eta_{i}}{h}\right)}
\end{aligned}
$$

$$
\begin{aligned}
& \text { until }\left(k_{3} k_{4} c|h| / 6\left(1-k_{3} k_{4} c|h|\right)\right)^{2^{j-1}}<|h| \text { and } \\
& \left|\eta_{i+1, j}-\eta_{i+1, j-1}\right| \leq \epsilon \text { are satisfied }
\end{aligned}
$$

c)

$$
\begin{array}{r}
\quad \eta_{i+1}=\eta_{i+1, j} \\
t_{i+1}=t_{i}+h .
\end{array}
$$

Let $m$ denote the least non-negative integer such that the conditions $\left(k_{3} k_{4} c|h| / 6\left(1-k_{3} k_{4} c|h|\right)\right)^{2^{m-1}}<|h|$ and $\left|\eta_{i+1, m}-\eta_{i+1, m-1}\right| \leq \epsilon$ are satisfied for a fixed $i$. 
As in Scheme III, the approximations $\eta_{i m}$ are the Newton's approximations and by conditions $(I V)-(V I)$, they are well defined, and the following theorem can be proved similarly.

Theorem 5 (Error estimate and Convergence of the Scheme IV): Let $e_{i}=\eta_{i}-y\left(t_{i}\right)$, $i=0,1,2, \ldots, n$. Then $\left|e_{i}\right| \leq \mathcal{N} h\left(e^{\alpha k_{1} /\left(1-k_{2}\right)}-1\right), i=0,1,2, \ldots, n$, where the constant $\mathcal{N}$ is same as in Theorem 4.

From Theorems 4 and 5, we note that the numerical Schemes III and IV are equivalent in the sense that in both the cases the approximations converge to the true solution at the same rate. Also it can be shown that the influence of the round-off errors in both the schemes are same.

The above schemes give approximate values of the solution in the interval $\left[t_{0}, t_{0}+\alpha\right]$. By changing $h$ to $-h$, the schemes can also be used to find approximate values of the solution in the interval $\left[t_{0}-\alpha, t_{0}\right]$. For this case, in the above discussions $h$ needs to be replaced by $|h|$ whenever $h$ appears in an inequality.

\section{COMPUTATION OF TIE SOLUTIONS OF SOME NSTD IVPs}

Example 1: Consider the NSTD IVP

$$
y^{\prime}=\frac{1}{7} e^{t-1}+\frac{2}{7} y+\frac{1}{14} y^{2} \cos y^{\prime}, y(0)=1 \text {. }
$$

Taking the domain $D=\left\{(t, y, z) \in R^{3}|| t|\leq 1| y-,1|\leq 1| z \mid, \leq 1\right\}$, it can be shown using theorem 1 , as in section 3 of [7], that the $I V P(25)$ has a unique solution existing on the interval $[-1,1]$.

a) Computation of the solution using Scheme I:

The solution of (25) is computed using Scheme I and the graphs of the (numerical) solution for various step sizes $h$ are given below.

SCHEME I

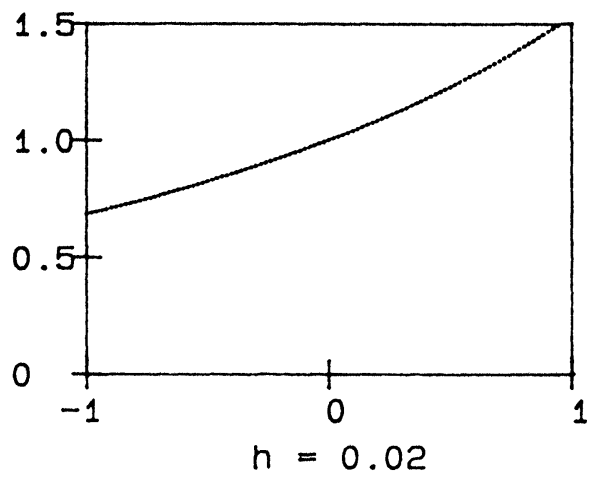

SCHEME I

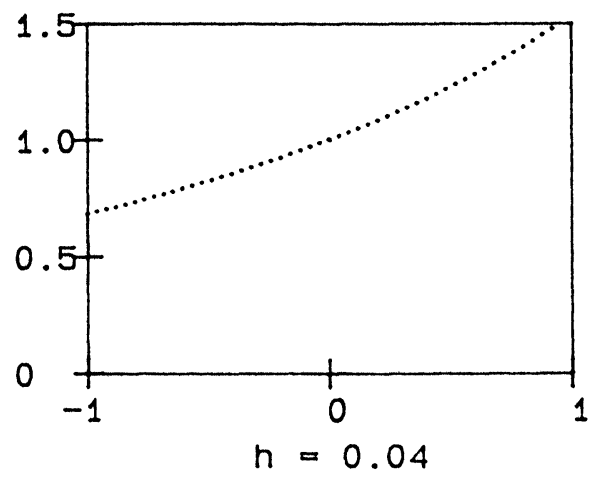


SCHEME I

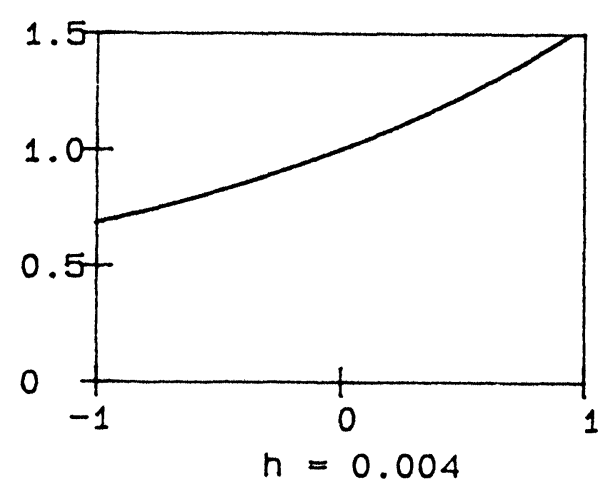

SCHEME I

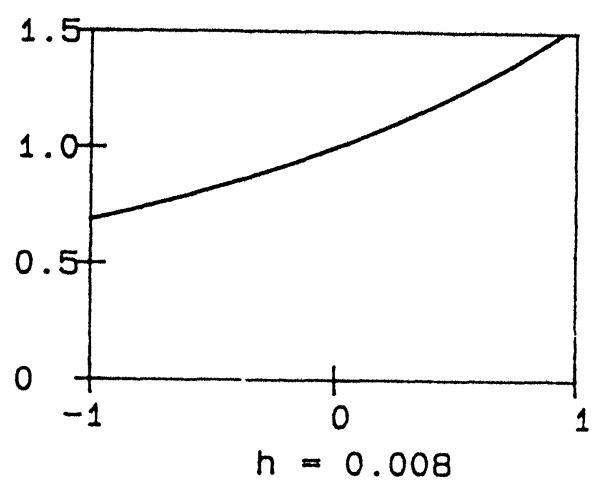

b) Computation of the solution using Scheme II:

The solution of (25) is computed using Scheme II and the graphs of the (numerical) solution for various step sizes $h$ are given below.

SCHEME II

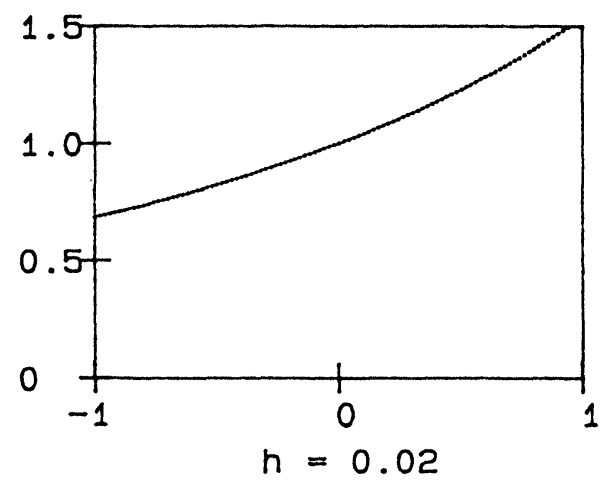

SCHEME II

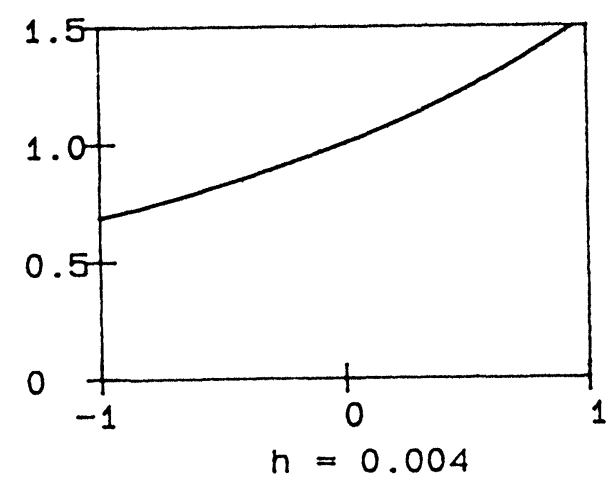

SCHEME II

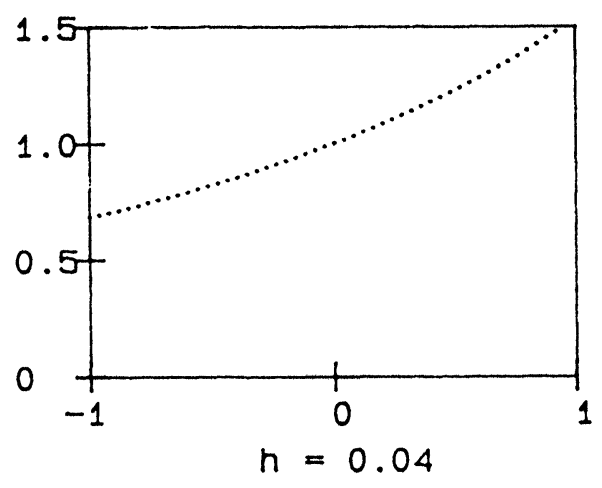

SCHEME II

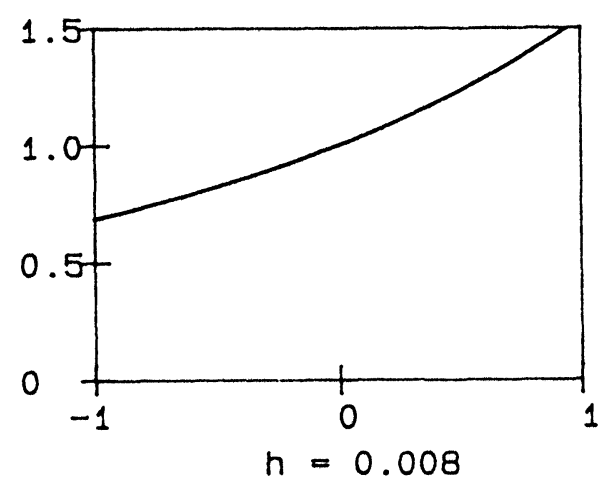


Example 2: Consider the NSTD IVP

$$
y^{\prime}=\frac{1}{28} e^{t-1}+\frac{1}{14} y+\frac{1}{14} y^{2} \cos y^{\prime}, y(0)=1 .
$$

Taking the same domain $D$ as in Example 1, it can be shown, using Theorem 1, that the IVP (26) has a unique solution existing on the interval $[-1,1]$.

c) Computation of the solution using Scheme III:

The solution of (26) is computed using Scheme III and the graphs of the (numerical) solutions for various step sizes $h$ are given below.

SCHEME III

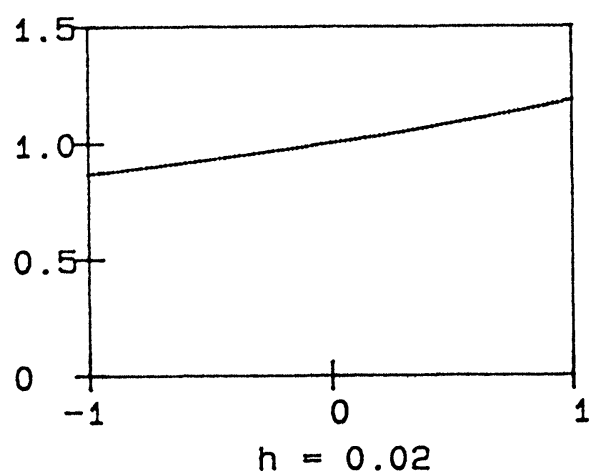

SCHEME III

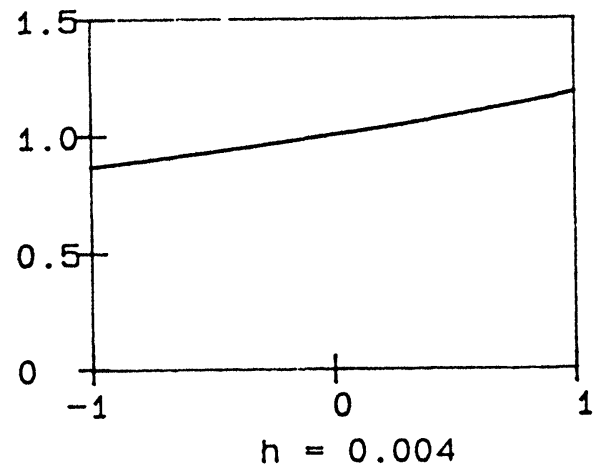

SCHEME III

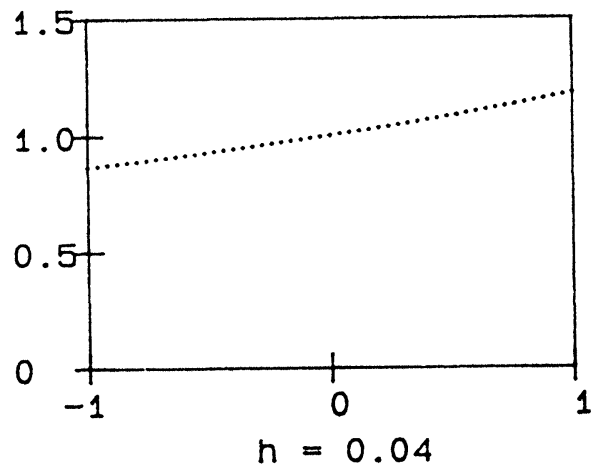

SCHEME III

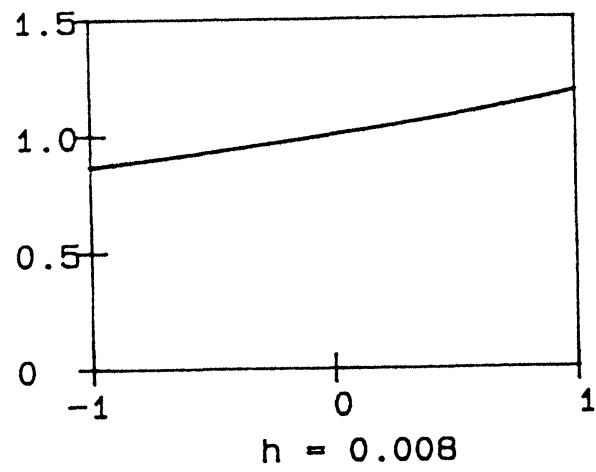


d) Computation of the solution using Scheme IV:

The solution of (26) is computed using Scheme IV and the graphs of the (numerical) solution for various step sizes are given below.

SCHEME IV

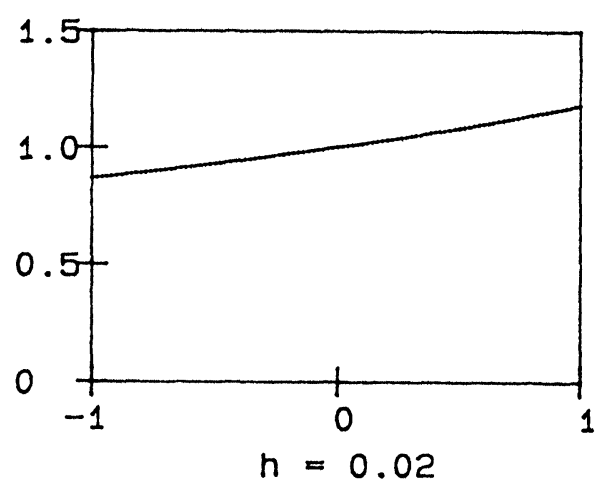

SCHEME IV

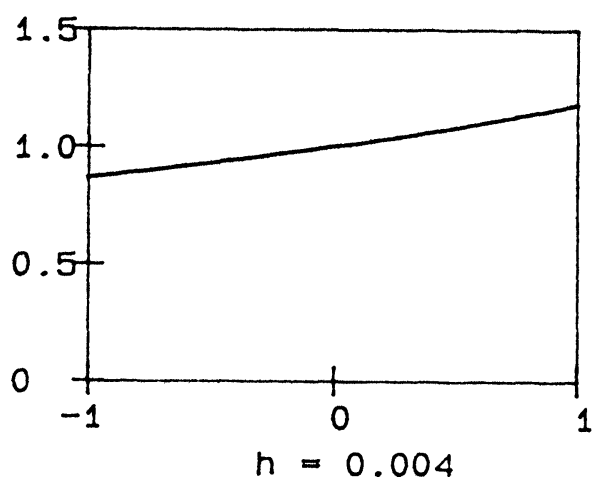

SCHEME IV

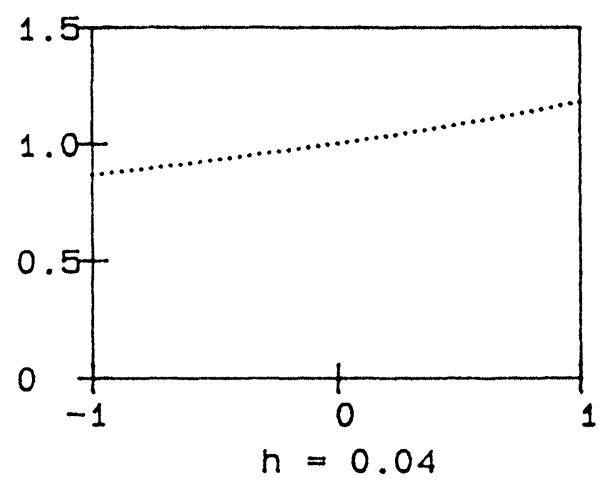

SCHEME IV

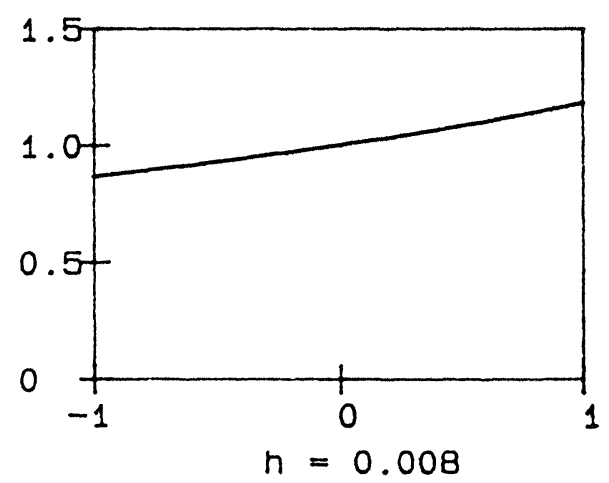

\section{ACKNOWLEDGEMENT}

The authors dedicate the work to the Chancellor of the Institute Bhagawan Sri Sathya Sai Baba. 


\section{REFERENCES}

[1] R.G. Bartle, "The Elements of Real Analysis", John Wiley, New York, 1976.

[2] H.T. Davis, "Introduction to Nonlinear Differential and Integral Equations", Dover Publications, 1962.

[3] E.L. Ince, “Ordinary Differential Equations”, Dover Publications, New York, 1956.

[4] M.A. Krasnosel'skii, et. al., “Approximate Solutions of Operator Equations”, Wolters Noordhoff Publishing, Groningen, 1972.

[5] H.B. Keller, “Numerical Methods for Two-Point Boundary Value Problems," Blaisdell Publishing Company, Waltham, 1968.

[6] J. Stoer and R. Bulirsch, "Introduction to Numerical Analysis", Springer-Verlag, New York, 1980.

[7] M. Venkatesulu and P.D.N. Srinivasu, "Solutions of nonstandard initial value problems for a first order ordinary differential equation", Journ. Appl. Math. Simul., Vol. II, No. 4, (1989), pp. 225-238. 


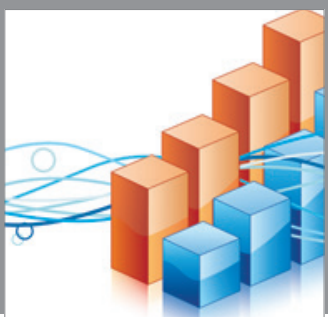

Advances in

Operations Research

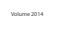

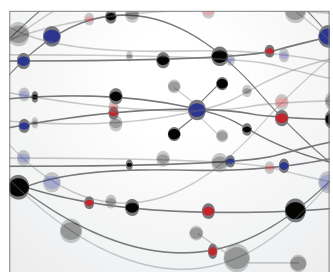

\section{The Scientific} World Journal
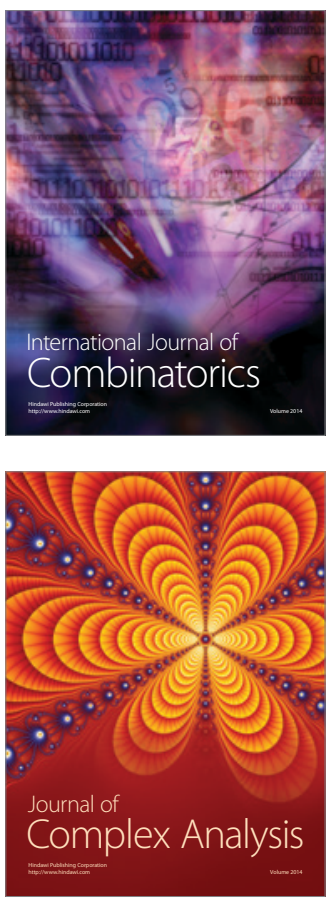

International Journal of

Mathematics and

Mathematical

Sciences
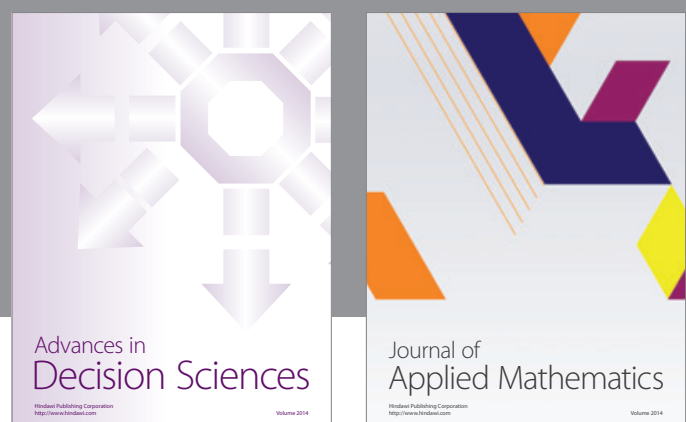

Journal of

Applied Mathematics
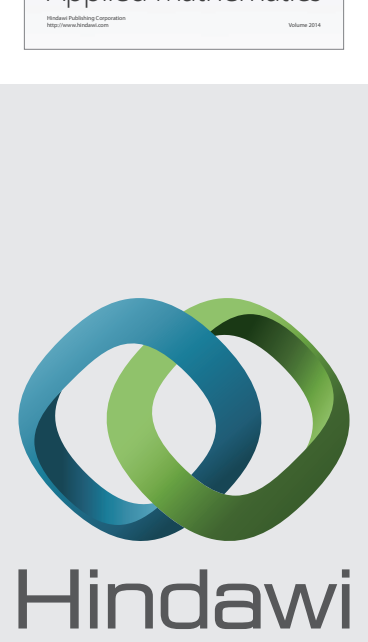

Submit your manuscripts at http://www.hindawi.com
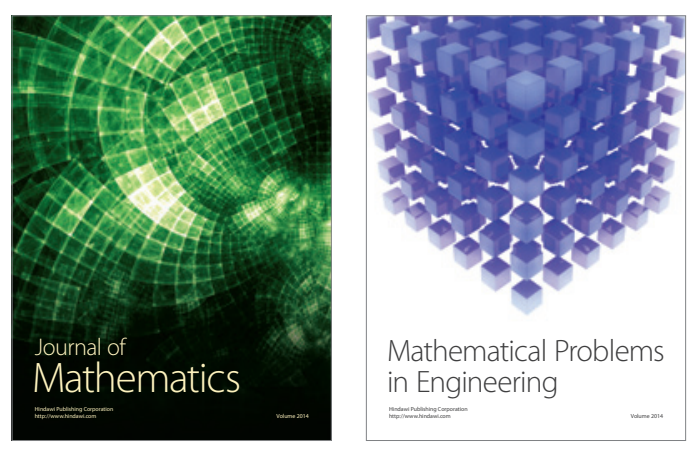

Mathematical Problems in Engineering
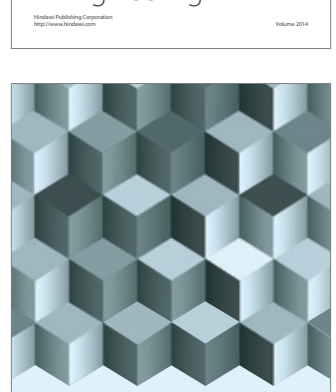

Journal of

Function Spaces
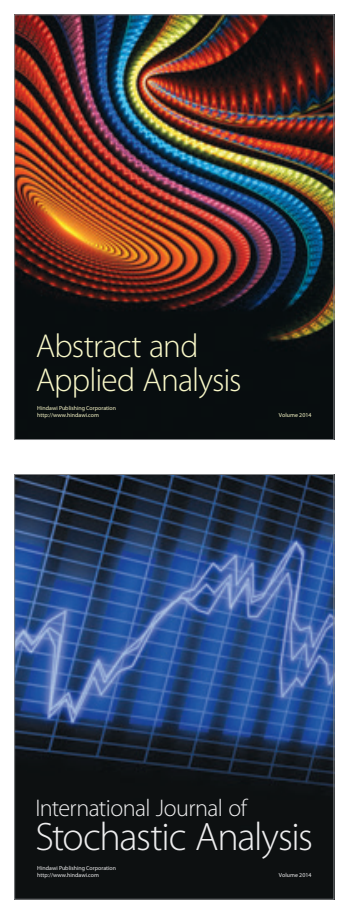

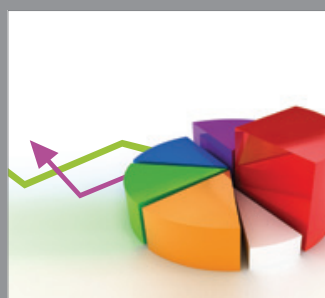

ournal of

Probability and Statistics

Promensencen
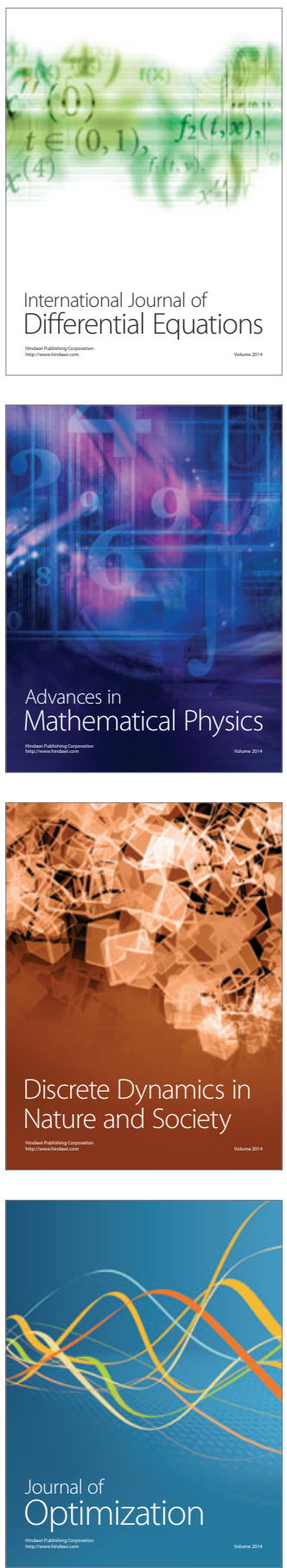